\title{
Characterization of Ultra Low-K Dielectric Materials by STEM EELS Elemental Mapping
}

Wayne W. Zhao, Michael Gribelyuk, and Jeremy D. Russell

Engineering Analysis - Physical, Technology Development \& Yield Engineering, GLOBALFOUNDRIES, Malta, New York, USA.

Various materials including species, which are sensitive to the electron beam, have been introduced into the Si-based wafer process and integration. This requires the development of the novel analytical techniques, which would be capable to evaluate these structures without causing the beam damage. For example, the ultra-low-k (ULK, $\mathrm{k}$ is the dielectric constant) $\mathrm{SiOC}(\mathrm{H})$ is one of such materials, which is used extensively in the semiconductor industry to enhance properties of the copper-based advanced back-end-of-line (BEOL) interconnects[1]. Control and further development of these films requires information about their elemental composition with high spatial resolution [2 6].

In the electron microscope the extent of the electron beam damage depends on the accelerating voltage, the electron beam density and the total electron dose. The electron beam density was 9.987 nA / angstrom $^{2}$, (calibrated with a Faraday cup). The electron probe size was 5 angstrom. The work has focused on the determination of the maximum dose, which would cause minimum electron beam damage of the $\operatorname{SiCO}(\mathrm{H})$ during analysis.

The electron energy loss spectroscopy (EELS) technique with the Gatan Quantum GIF was used for the 2-D mapping of the elemental composition of $\mathrm{SiCO}(\mathrm{H})$, as EELS is sensitive to the light atomic weight elements and provides the minimum readout time [7 8]. A series of analysis was performed whereby the acquisition time at each pixel was varied. The size of the map and all other parameters were kept constant. We used neighboring identical structures of the $\operatorname{SiCO}(\mathrm{H})$ in the same TEM sample in each analysis.

Demonstrated here is one example of the analysis of the $\operatorname{SiCO}(\mathrm{H})$ material, which was used to build a SRAM device. Figure 1 shows a STEM image and the individual EELS elemental maps acquired by Digiscan Spectrum Imaging [9], with a pixel time of 10 millisecond. The individual elemental line profiles of $\mathrm{Si}, \mathrm{C}, \mathrm{O}$ were extracted from each analysis and compared. They were used to determine the maximum dose, which does not cause the electron beam damage. In addition, the same maps were acquired by the TIA acquisition system. Since the readout time in TIA is longer compared to that when the Gatan Digiscan system is used, we reduced the acquisition time so that the dose is kept the same. The maps acquired by the Digiscan and TIA using the same dose were compared to evaluate the effect of the reduced acquisition time on the noise in the extracted elemental profiles. (Thanks go to Cynthia Martin for her excellent supports in preparing TEM samples nicely.)

References:

[1] Maex K. et al.: J. Appl. Phys. Vol. 93, (2003), pp. 8793-8800.

[2] W. Zhao, et al., Microscopy \& Microanalysis, Vol. 20 (Supplement 3), (2014), pp.362 363.

[3] W. Zhao, M. Gribelyuk, et al., Proc. $38^{\text {th }}$ International Symposium for Testing and Failure Analysis, (2012), pp. 347 355. 
[4] W. Zhao, et al., Microscopy \& Microanalysis, Vol. 20 (Supplement 3), (2014), pp.1000 1001.

[5] W. Zhao, et al., Microscopy \& Microanalysis, Vol. 19 (Supplement 2), (2013), pp.902 903.

[6] W. Zhao, Symp. Proc. the Material Research Society, 2002 Fall Meeting, (2002), Vol. 738, pp. G7.15.1 6.

[7] R. Leapman and J. Hunt, Microscopy, Microanalysis, Microstructure, Vol. 2, (1991) pp. 231-244.

[8] H. Harrach, et al., Microscopy \& Microanalysis, Vol. 16 (Supplement 2), (2010), pp.1312 1313.

[9] http://www.gatan.com/products/sem-imaging-spectroscopy/digiscan-ii-system.
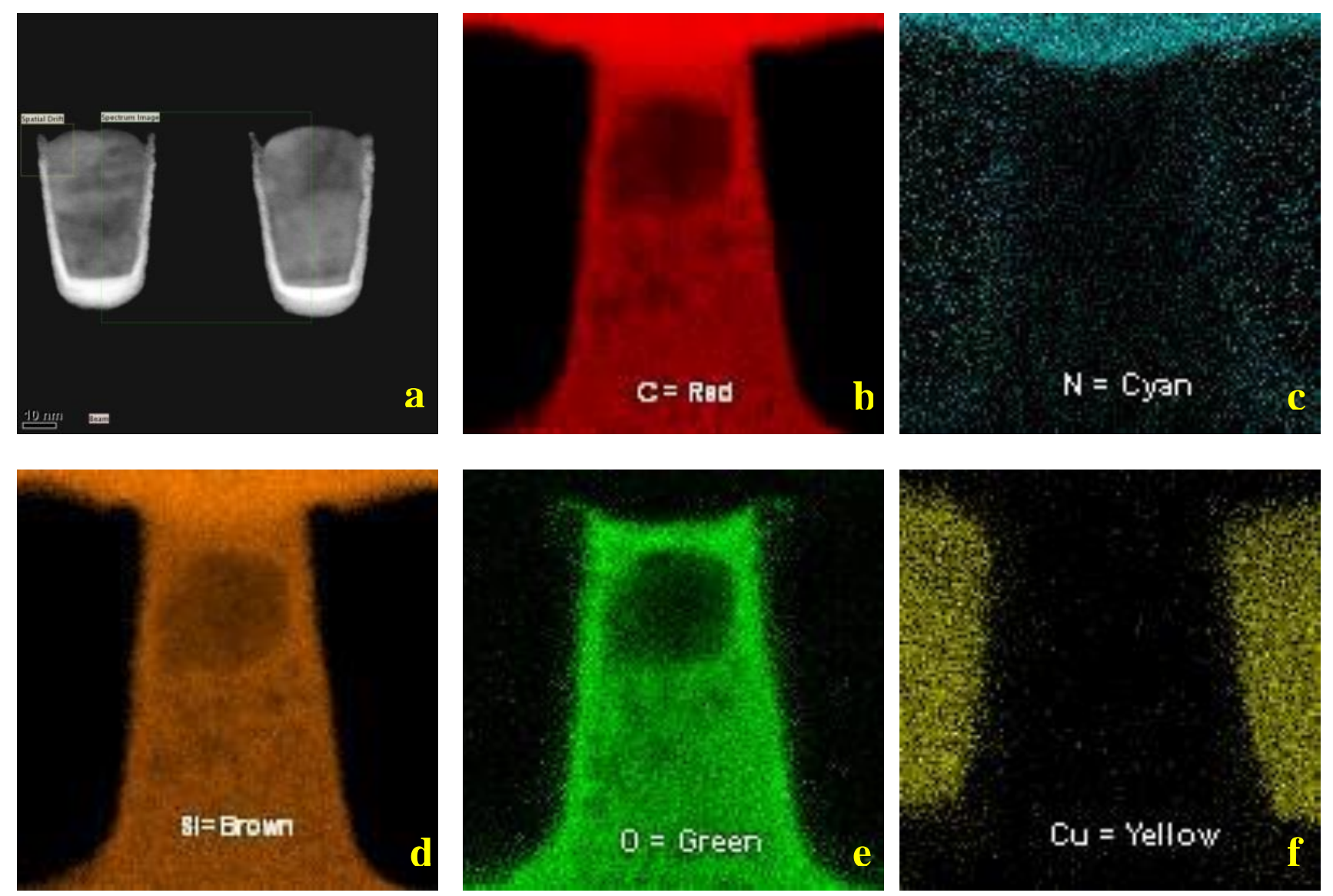

Figure 1. STEM EELS elemental mapping by Digiscan Spectrum Imaging, (a) HAADF-STEM image, and STEM-EELS elemental mapping by Digiscan spectrum imaging; (b) C (red); (c) N (cyan); (d) Si (brown); (e) $\mathrm{O}$ (green); and (f) $\mathrm{Cu}$ (yellow). 